\title{
抗褐变马铃薯品种(系)鉴定与笁选
}

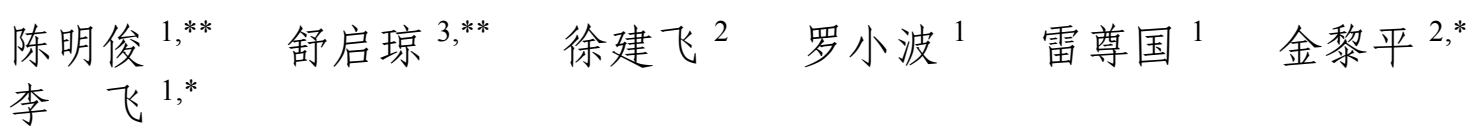

\footnotetext{
1 贵州省农业科学院马铃薯研究所 / 国家马铃薯改良中心贵州分中心, 贵州贵阳 $550006 ;^{2}$ 中国农业科学院蔬菜花卉研究所, 北京 $100081 ;^{3}$ 贵州师范大学生命科学学院, 贵州贵阳 550000
}

摘 要: 马铃薯块茎易受到损伤引发褐变, 褐变会对其营养、感官品质和安全性造成影响。不同马铃薯品种材料抗 褐变能力存在差异, 本研究拟测定 27 份不同马铃薯种质材料的多酚氧化酶活性、褐变强度、褐变指数和者后褐变 4 个褐变相关指标, 综合分析以实现对不同马铃薯品种(系)抗褐变能力的鉴定与篮选。结果表明, 27 份材料可分为 4 种 类型, 第 1 类高抗褐变, 包括 09306-82 和 13041-52, 它们 4 项指标均能满足抗褐变材料的要求; 第 2 类抗褐变材料, 包括延薯 4 号、Q8、12-1、春薯 3 号、19-1、14018-142、大西洋、龙薯 12 号、威芋 5 号、丽薯 6 号、春薯 5 号和 早大白, 它们的褐变相关指标存在个别明显不足; 第 3 类中度褐变材料, 包括 09001-136、中薯 5 号、龙薯 4 号、东 农 310、青薯 9 号、15-1、云薯 505、BF006、闽薯 1 号和黔芋 8 号, 其抗褐变能力弱; 第 4 类是易褐变材料, 包括春 薯 6 号和费乌瑞它, 它们不具有抗褐变能力或抗褐变能力非常差。篮选的高抗褐变和抗褐变品种(系)可以作为鲜食抗 褐变马铃薯育种材料, 为马铃薯净菜加工提供品种支撑, 易褐变材料可用于马铃薯褐变机制的基础性研究。

关键词: 马铃薯; 抗褐变; 多酚氧化酶; 褐化指数; 褐化强度; 者后变褐

\section{Identification and selection for anti-browning potato varieties (lines)}

\author{
CHEN Ming-Jun ${ }^{1, * *}$, SHU Qi-Qiong ${ }^{3, * *}$, XU Jian-Fei ${ }^{2}$, LUO Xiao-Bo ${ }^{1}$, LEI Zun-Guo ${ }^{1}$, JIN Li-Ping ${ }^{2, *}$, and LI \\ $\mathrm{Fei}^{1, *}$ \\ ${ }^{1}$ Institute of Potato, Guizhou Academy of Agricultural Sciences, Guiyang 550006, Guizhou, China; ${ }^{2}$ Institute of Vegetables and Flowers, Chinese \\ Academy of Agricultural Sciences, Beijing 100081, China; ${ }^{3}$ College of Life Sciences, Guizhou Normal University, Guiyang 550000, Guizhou, China
}

\begin{abstract}
Potato tuber is easy to be damaged and caused browning, which will affect its nutrition, sense quality and safety. Different potato varieties have different resistances to browning. In this study, 27 different potato germplasm resources,were used to measure four indexes including polyphenol oxidase (PPO), browning degree (BD), browning index and after cooking darkening (ACD), for indentifying and selecting anti-browning potato varieties (lines). The 27 materials could be divided into four groups. High anti-browning, group including 09306-82 and 13041-52 had high resistance to browning. Anti-browning group, included Yanshu 4, Q 8, 12-1, Chunshu 3, 19-1, 14018-142, Atlantic, Longshu 12, Weiyu 5, Lishu 6, Chunshu 5, and Zaodabai, had some obvious deficiencies in the related anti-browning indicators. Medium anti-browning group, including 09001-136, Zhongshu 5, Longshu 4, Dongnong 310, Qingshu 9, 15-1, Yunshu 505, BF006, Minshu 1, and Qianyu 8, had poor resistance to browning. Easy browning group, containing Chunshu 6 and Favorita, had very weak resistance to browning. The selected varieties (lines) with very high or high resistance to browning can be used in potato breeding for browning resistance, providing variety support for potato processing industry, and the easy browning materials can be used to study the browning mechanism of potato.
\end{abstract}

Keywords: potato; anti-browning; PPO; browning index; browning degree; after cooking darkening

\footnotetext{
本研究由国家重点研发计划子课题优质多抗适应性强马铃薯新品种培育(2017YFD0101905)和贵州省现代农业国际科技合作基地(黔 科合平台人才[2019]5804 号)资助。

This study was supported by the Sub-Project of the National Key Research and Development Plan (Cultivation of New Potato Varieties with High Quality, Multi Resistance and Strong Adaptability of China, 2017YFD0101905), and the Modern Agriculture International Science and Technology Cooperation Base of Guizhou (the Science and Technology Cooperation Talent Platform of Guizhou [2019] 5804).

*通信作者(Corresponding authors): 金黎平, E-mail: jinliping@caas.cn; 李飞, E-mail: gzlfei@sina.com

** 同等贡献(Contributed equally to this work)

第一作者联系方式: 陈明俊, E-mail: 18286033948@163.com; 舒启琼, E-mail: shuqiqiong@163.com

Received (收稿日期): 2019-11-25; Accepted (接受日期): 2020-03-24; Published online (网络出版日期): 2020-04-26.

URL: http://kns.cnki.net/kcms/detail/11.1809.s.20200426.1236.002.html
} 
马铃薯 (Solanum tuberosum L.)作为世界上第四 大粮食作物, 是全球近 10 亿人的主食 ${ }^{[1]}$ 。马铃薯褐 变会对其营养、感官和食味品质造成影响, 导致商 品价值降低 ${ }^{[2]}$, 阻碍其加工业和产业的快速发展。马 铃薯褐变包括酶促褐变与非酶褐变, 在加工过程中 以酶促褐变为主 ${ }^{[3]}$, 酶促褐变发生在块茎受到机械 损伤时, 有氧气存在的条件下, 氧化酶将酚类物质 逐步氧化成醌类物质, 进而与氨基酸和蛋白质等聚 合生成深褐色或黑色的聚合物 ${ }^{[4]}$ 。生成的聚合物不 溶于水, 人体很难将其消化吸收, 导致马铃薯营养 的丢失, 同时存在安全性问题 ${ }^{[5]}$ 。多酚氧化酶 (polyphenol oxidase, PPO)是参与酶促褐变最主要的 酶类 ${ }^{[6]}$, 抑制 PPO 活性有利于降低褐变的发生 ${ }^{[7-8]}$ 。 此外, 多酚是天然的抗氧化剂, 对预防癌症和心血 管疾病具有潜在的积极作用，因而多酚类化合物被 氧化对人类食用是非常不利的 ${ }^{[9]}$ 。非酶褐变包括焦 糖化反应、美拉德反应、抗坏血酸的氧化分解以及 酚类的氧化缩合现象 ${ }^{[10]}$, 典型特征是不需要酶的参 与, 主要是化学反应导致果肉和果皮的褐变。

国内外学者对褐变研究有很多, 但是多为对褐

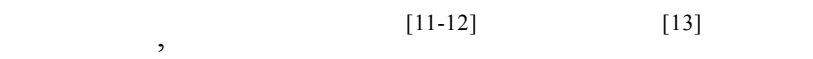
工程 ${ }^{[14-15]}$ 改良。这些方法对褐变的抑制能起到一些 积极作用, 但也加大成本投入, 同时存在安全性问 题 ${ }^{[16]}$ 。随着褐变机制研究的不断深入, 人们逐渐认 识到选育抗褐变品种才能从源头解决或减轻农产品 在陉藏和加工中的褐变问题 ${ }^{1]}$ 。常见的马铃薯栽培品 种以四倍体为主, 不同马铃薯材料抗褐变能力存在 明显差异 ${ }^{[17-18]}$ 。本研究拟对从国内收集的 27 份优质 马铃薯材料抗褐变能力进行鉴定篮选, 篮选出的高 抗褐变和抗褐变品种(系)将为培育鲜食抗褐变马铃薯 品种提供育种材料, 为马铃薯净菜加工提供品种支 撑, 易褐变材料可用于马铃薯褐变机制的研究。

\section{1 材料与方法}

\section{1 试验材料}

27 份供试材料为近年新选育的马铃薯鲜食或加 工新品种(系), 具有较好的品质和抗性, 且部分品种 为当前生产上的主栽品种, 均由贵州省农业科学院 马铃薯研究所收集提供, 于 2019 年 2 月种植于贵州 省农业科学院内试验基地, 并于 7 月收获。从收获 的每份马铃薯试验材料中, 挑选薯型大小均匀、块 茎新鲜、无病虫害、无明显机械损伤、无青皮的 10 个马铃薯块茎用于试验。

\section{2 多酚氧化酶(PPO)活性测定}

参考庞学群等 ${ }^{[19]}$ 方法测定 PPO 活性。先制备粗 酶提取液, 然后用酶标仪测定 PPO 活性, 反应体系 为 $0.1 \mathrm{~mol} \mathrm{~L}^{-1}$ 磷酸缓冲液 $(\mathrm{pH} 7.0) 0.1 \mathrm{~mL} 、 0.5 \mathrm{~mol}$ $\mathrm{L}^{-1}$ 邻苯二酚 $0.05 \mathrm{~mL} 、 0.1 \% \mathrm{SDS} 0.025 \mathrm{~mL} 、 \mathrm{PPO}$ 粗 酶液 $0.05 \mathrm{~mL}$ 。以磷酸缓冲液为对照, 加入酶液后, 在 $25^{\circ} \mathrm{C}$ 条件下反应 $15 \mathrm{~s}$ 时开始记录反应体系在波长 $420 \mathrm{~nm}$ 处吸光值, 作为初始值, 然后每隔 $10 \mathrm{~s}$ 记录 1 次, 连续测定, 获取 6 个点的数据。每个样品重复 3 次。以每分钟 $\mathrm{OD}_{420}$ 变化 0.001 作为一个酶活力单位 (U), 以 $\mathrm{U} \mathrm{g}^{-1} \mathrm{FW}$ 表示。

\section{3 褐变强度测定}

参照李云山等 ${ }^{[20]}$ 方法测定褐变强度。以去离子 水作为对照, 首先将材料处理后置 $30^{\circ} \mathrm{C}$ 水浴中 $20 \mathrm{~min}$, 取部分匀浆 $10,111 \times g$ 离心 $10 \mathrm{~min}$, 取上清 液测定 $420 \mathrm{~nm}$ 处吸光值。剩余部分于 $4^{\circ} \mathrm{C}$ 放置 $24 \mathrm{~h}$ 后 $10,111 \times g$ 离心 $10 \mathrm{~min}$, 再取上清液测定 $420 \mathrm{~nm}$ 处 吸光值。

\section{4 褐变指数测定}

参考王清等 ${ }^{[21]}$ 方法测定褐变指数。从每份马铃 薯材料中挑选 3 个大小一致的块茎, 自来水清洗后 用吸水纸擦干将块茎平均切开, 再切出相似大小 $0.5 \mathrm{~cm}$ 厚的薯片各 3 片, 暴露在室温 $\left(25^{\circ} \mathrm{C}\right)$ 条件下, 并在切后 $0 、 0.5 、 1 、 2 、 3 、 4 、 6 、 8 、 12$ 和 $24 \mathrm{~h}$ 观察情况并在统一条件下照相, 计算块茎的酶促褐 变指数。依据褐化面积记录褐变等级。0 级是无褐 变; 1 级是褐变面积 $25 \%$ 以内; 2 级是褐变面积在 25\%至 50\%之间；3 级是褐变面积在 50\%至 75\%； 4 级是褐变面积达到 $75 \%$ 及以上。褐变指数越高则褐 变程度越重。

褐化指数 $=\sum[$ (褐化级别 $\times$ 该级别块茎数 $) /$ (最高级数 $\times$ 检查的总块茎数) $] \times 100 \%$ 。

\section{5 者后变褐(After cooking darkening, ACD)测定}

参照王唯俊 ${ }^{[2]}$ 测定煮后变褐( $\left.\mathrm{ACD}\right)$ 。去皮蒸煮 过程中保持块茎在水面下, 避免酶促变色。将蒸煮 过的块茎在自然条件下放置 $24 \mathrm{~h}$ 后, 与标准色比对 评价。共分为 6 个等级, 从 4 级到 9 级, 9 级最高, 完 全没有变色; 4 级最低, 已变成深灰或黑色。

\section{2 结果与分析}

\section{1 主要农艺性状}

27 份马铃薯材料的主要农艺性状如表 1 , 马铃 薯的薯肉颜色有黄色、淡黄色和白色。 
表 1 马铃薯品种(系)的主要农艺性状

Table 1 Main agronomic traits of potato varieties (lines)

\begin{tabular}{|c|c|c|c|c|c|c|c|}
\hline $\begin{array}{l}\text { 编号 } \\
\text { No. }\end{array}$ & $\begin{array}{c}\text { 品种(系) } \\
\text { Variety (line) }\end{array}$ & $\begin{array}{c}\text { 株高 } \\
\text { Plant height } \\
(\mathrm{cm})\end{array}$ & $\begin{array}{c}\text { 花色 } \\
\text { Flower color }\end{array}$ & $\begin{array}{c}\text { 薯形 } \\
\text { Tuber shape }\end{array}$ & $\begin{array}{c}\text { 主茎数 } \\
\text { Main stem } \\
\text { number }\end{array}$ & $\begin{array}{c}\text { 肉色 } \\
\text { Flesh color }\end{array}$ & $\begin{array}{l}\text { 芽眼深度 } \\
\text { Eye depth }\end{array}$ \\
\hline 1 & 大西洋 Atlantic & 62 & 淡紫色 Lilac & 卵圆形 Oval & 3 & 白色 White & 浅 Shallow \\
\hline 2 & 黔芋 8 号 Qianyu 8 & 60 & 白色 White & 椭圆形 Oblong & 8 & 黄色 Yellow & 浅 Shallow \\
\hline 3 & 中薯 5 号 Zhongshu 5 & 61 & 白色 White & 扁圆形 Oblateness & 4 & 淡黄 Flaxen & 浅 Shallow \\
\hline 4 & 闽薯 1 号 Minshu 1 & 43 & 白色 White & 长圆形 Obround & 4 & 淡黄 Flaxen & 浅 Shallow \\
\hline 5 & 09306-82 & 62 & 紫色 Purple & 长圆形 Obround & 5 & 黄色 Yellow & 浅 Shallow \\
\hline 6 & 09001-136 & 65 & 白色 White & 卵圆形 Oval & 4 & 黄色 Yellow & 浅 Shallow \\
\hline 7 & 费乌瑞它 Favorita & 48 & 蓝紫色 & 椭圆形 Oblong & 4 & 黄色 Yellow & 浅 Shallow \\
\hline 8 & 龙薯 6 号 Longshu 6 & 49 & $\begin{array}{l}\text { Bluish violet } \\
\text { 淡紫色 Lilac }\end{array}$ & 椭圆形 Oblong & 4 & 黄色 Yellow & 浅 Shallow \\
\hline 9 & 龙薯 12 号 Longshu 12 & 51 & 淡紫色 Lilac & 扁圆形 Oblateness & 3 & 白色 White & 浅 Shallow \\
\hline 10 & $19-1$ & 48 & 白色 White & 椭圆形 Oblong & 3 & 黄色 Yellow & 中 Medium \\
\hline 11 & 延薯 4 号 Yanshu 4 & 49 & 白色 White & 圆形 Roundness & 3 & 黄色 Yellow & 中 Medium \\
\hline 12 & 春薯 6 号 Chunshu 6 & 54 & 白色 White & 椭圆形 Oblong & 5 & 黄色 Yellow & 浅 Shallow \\
\hline 13 & $12-1$ & 66 & 白色 White & 椭圆形 Oblong & 4 & 黄色 Yellow & 浅 Shallow \\
\hline 14 & $14018-142$ & 57 & 紫色 Purple & 椭圆形 Oblong & 6 & 黄色 Yellow & 中 Medium \\
\hline 15 & 威芋 5 号 Weiyu 5 & 61 & 白色 White & 椭圆形 Oblong & 5 & 黄色 Yellow & 浅 Shallow \\
\hline 16 & 东农 310 Dongnong 310 & 60 & 淡紫色 Lilac & 扁圆形 Oblateness & 4 & 白色 White & 浅 Shallow \\
\hline 17 & 春薯 3 号 Chunshu 3 & 55 & 白色 White & 圆形 Roundness & 4 & 白色 White & 浅 Shallow \\
\hline 18 & 龙薯 4 号 Longshu 4 & 47 & 紫色 Purple & 长圆形 Obround & 4 & 黄色 Yellow & 浅 Shallow \\
\hline 19 & $13041-52$ & 42 & 白色 White & 卵圆形 Oval & 5 & 淡黄 Flaxen & 浅 Shallow \\
\hline 20 & 云薯 505 Yunshu 505 & 59 & 白色 White & 扁圆形 Oblateness & 7 & 白色 White & 浅 Shallow \\
\hline 21 & BF006 & 64 & 白色 White & 长圆形 Obround & 7 & 黄色 Yellow & 浅 Shallow \\
\hline 22 & $15-1$ & 50 & 紫色 Purple & 椭圆形 Oblong & 6 & 黄色 Yellow & 浅 Shallow \\
\hline 23 & 早大白 Zaodabai & 64 & 白色 White & 长圆形 Obround & 2 & 白色 White & 浅 Shallow \\
\hline 24 & 春薯 5 号 Chunshu 5 & 60 & 白色 White & 扁圆形 Oblateness & 5 & 白色 White & 浅 Shallow \\
\hline 25 & Q8 & 51 & 淡紫色 Lilac & 椭圆形 Oblong & 5 & 黄色 Yellow & 浅 Shallow \\
\hline 26 & 青薯 9 号 Qingshu 9 & 65 & 浅红色 Light red & 椭圆形 Oblong & 3 & 黄色 Yellow & 浅 Shallow \\
\hline 27 & 丽薯 6 号 Lishu 6 & 62 & 白色 White & 椭圆形 Oblong & 5 & 白色 White & 浅 Shallow \\
\hline
\end{tabular}

\section{2 马铃薯品种(系)的 PPO 活性}

PPO 在马铃薯酶促褐变中发挥重要作用, 本研 究将 PPO 活性在 $300 \mathrm{U} \mathrm{g}^{-1}$ 以内作为篮选抗褐变预选 材料标准, 通过对不同马铃薯品种(系)的 PPO 活性 测定, 发现不同品种(系)多酚氧化酶活性存在明显 差异(图 1), PPO 活性在 $200 \mathrm{U} \mathrm{g}^{-1}$ 以内的马铃薯材料 有 9 份, 其中 PPO 活性最低的是 13041-52, 仅为 90 $\mathrm{U} \mathrm{g}^{-1}$; PPO 活性在 $200 \mathrm{U} \mathrm{g}^{-1}$ 至 $300 \mathrm{U} \mathrm{g}^{-1}$ 以内的材料 有 7 份, 在 $300 \mathrm{U} \mathrm{g}^{-1}$ 到 $500 \mathrm{U} \mathrm{g}^{-1}$ 之间的材料有 4 份; PPO 活性高于 $500 \mathrm{U} \mathrm{g}^{-1}$ 的材料有 7 份, 其中丽薯 6 号最高, 是 13041-52 的 11.7 倍。符合要求的预选材
料有 16 份。

\section{3 马铃薯品种(系)的褐变强度}

褐变强度测定是将马铃薯块茎组织充分研磨, 使 PPO 和酚类物质最大程度地相遇发生褐变反应。 由图 2 可知, $\mathrm{Q} 8$ 褐变强度最小, 为 0.093 , 早大白初 始值最大, 是最小值的 2.82 倍; 在 $4^{\circ} \mathrm{C}$ 放置 $24 \mathrm{~h}$ 后 有所上升, 09306-82 最低值是 0.15, 19-1 褐变强度值 最高, 为 0.688 , 是 09306-82 的 4.59 倍; 这个过程中 褐变强度变化范围是 $0.044 \sim 0.471$, 相差 9.7 倍。将 马铃薯材料匀浆后 $4^{\circ} \mathrm{C}$ 放置 $24 \mathrm{~h}$, 其褐变强度在 0.3 以内匀浆呈现白色或者乳白色, 褐变强度在 0.300 


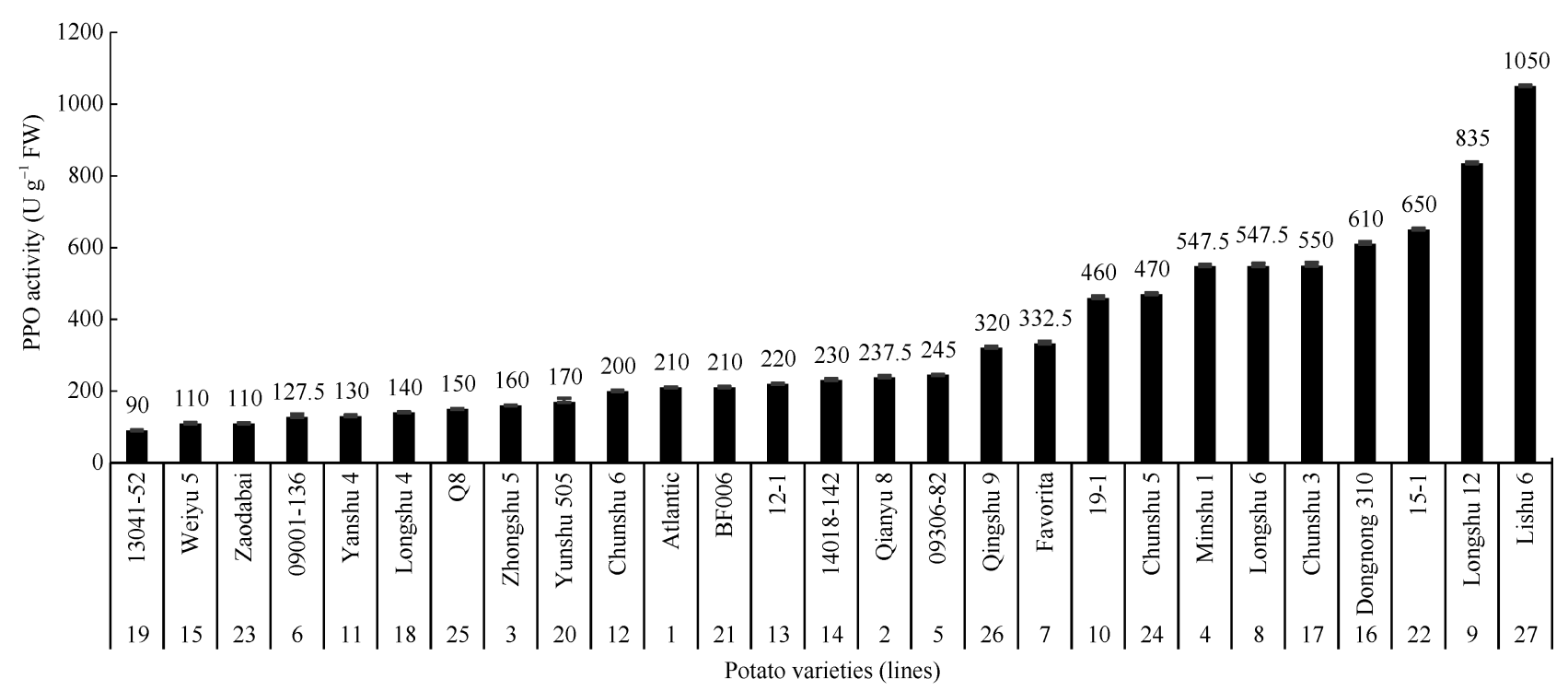

图 1 马铃薯品种(系)的 PPO 活性

Fig. 1 PPO activity of potato varieties (lines)

图中误差线为 3 个重复值的标准差。The error line was the standard deviation of three repeated values.

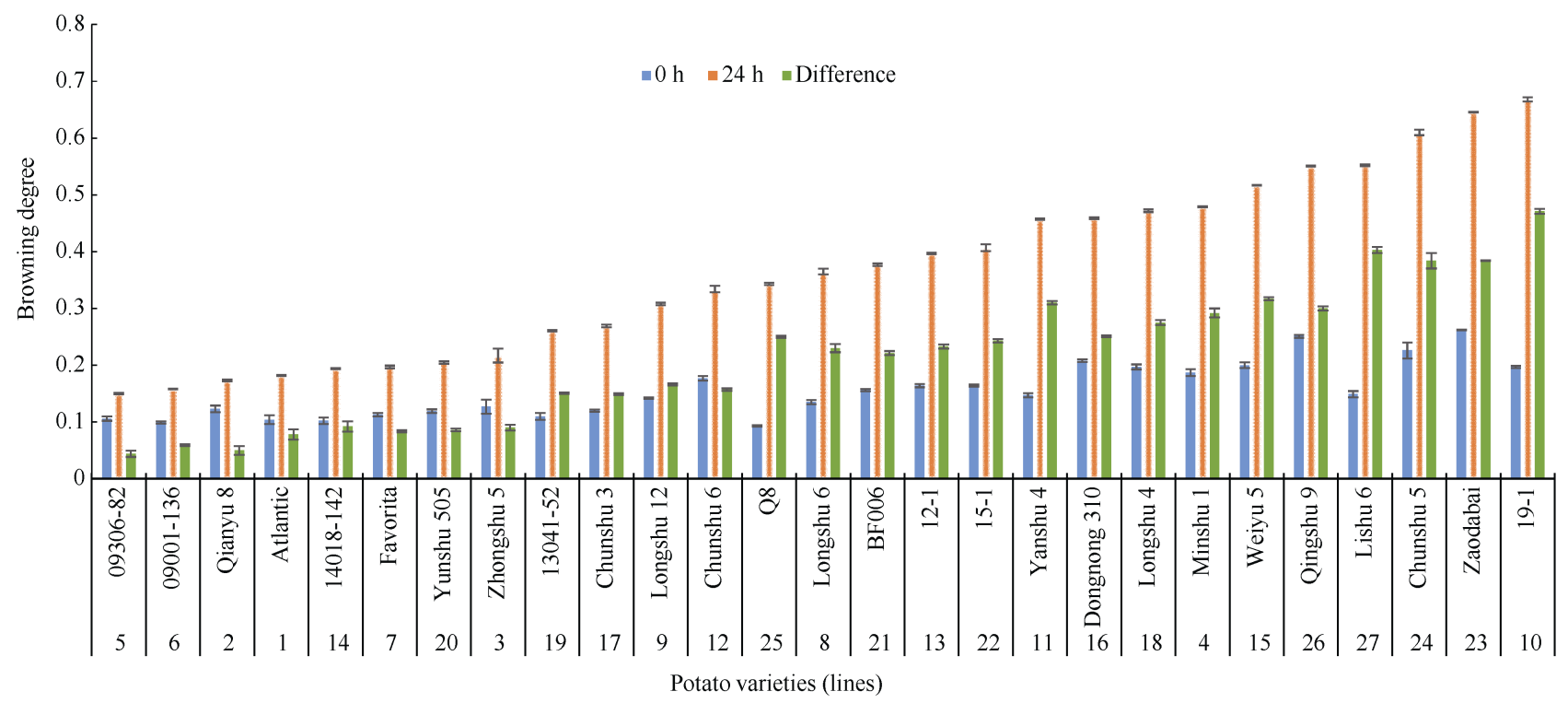

图 2 马铃薯品种(系)的褐变强度

Fig. 2 Browning degree of potato varieties (lines)

图中误差线为 3 个重复值的标准差。The error line was the standard deviation of three repeated values.

0.459 之间匀浆颜色为橘红色或者深灰色, 褐变强度 高于 0.459 匀浆就表现为黑色, 颜色随着褐变强度 增加而加深。

从 27 份材料中, 将初始褐变强度小于 0.15 , 变 化值不超过 0.2 的材料篮选出来, 共计 11 份材料, 作为抗褐变马铃薯预选材料(表 2)。

\section{4 马铃薯品种(系)的切片观察和褐变指数}

马铃薯品种(系)的切片观察结合褐变指数分级
可以直观地体现马铃薯的褐变情况。由图 3 可知, 费 乌瑞它、春薯 6 号和云薯 505 切片不足 $0.5 \mathrm{~h}$ 就开始 发生褐变, 在 $0.5 \sim 1 \mathrm{~h}$ 和 1 3 h 开始发生褐变的材料 各有 5 份, 在 $3 \sim 6 \mathrm{~h}$ 开始发生褐变的材料有 9 份, 在 6 8 h 开始发生褐变的材料有延薯 4 号和春薯 3 号, 在 8 12 h 开始发生褐变的有 13041-52、Q8 和丽薯 6 号。图 4 表明, 褐变指数 $25 \%$ 以内的有 09306-82、 延薯 4 号、19-1、13041-52、春薯 3 号和 Q8, 09306-82 
表 2 抗褐变马铃薯预选材料的褐变强度

Table 2 Primary selection of browning degree in anti-browning potato varieties (lines)

\begin{tabular}{clccc}
\hline 编号 & \multicolumn{1}{c}{$\begin{array}{c}\text { 品种(系) } \\
\text { No. }\end{array}$} & \multicolumn{3}{c}{ 褐变强度 Browning degree } \\
\cline { 3 - 5 } 5 & Variety (line) & $30^{\circ} \mathrm{C} / 20 \mathrm{~min}$ & $4^{\circ} \mathrm{C} / 24 \mathrm{~h}$ & 变化 Difference \\
\hline 2 & 黔芋 8 号 Qianyu 8 & $0.106 \pm 0.0040$ & $0.150 \pm 0.0015$ & $0.044 \pm 0.0055$ \\
6 & 09001 -136 & $0.123 \pm 0.0023$ & $0.173 \pm 0.0006$ & $0.050 \pm 0.0015$ \\
1 & 大西洋 Atlantic & $0.099 \pm 0.0059$ & $0.158 \pm 0.0015$ & $0.059 \pm 0.0076$ \\
7 & 费乌瑞它 Favorita & $0.104 \pm 0.0078$ & $0.182 \pm 0.0012$ & $0.078 \pm 0.0087$ \\
20 & 云薯 505 Yunshu 505 & $0.113 \pm 0.0057$ & $0.197 \pm 0.0006$ & $0.084 \pm 0.0090$ \\
3 & 中薯 5 号 Zhongshu 5 & $0.119 \pm 0.0032$ & $0.205 \pm 0.0025$ & $0.086 \pm 0.0021$ \\
14 & 14018-142 & $0.127 \pm 0.0031$ & $0.217 \pm 0.0023$ & $0.090 \pm 0.0023$ \\
17 & 春薯 3 号 Chunshu 3 & $0.102 \pm 0.0123$ & $0.194 \pm 0.0123$ & $0.092 \pm 0.0050$ \\
19 & 13041 -52 & $0.120 \pm 0.0062$ & $0.269 \pm 0.0012$ & $0.149 \pm 0.0012$ \\
9 & 龙薯 12 号 Longshu 12 & $0.110 \pm 0.0020$ & $0.261 \pm 0.0021$ & $0.151 \pm 0.0015$ \\
\hline
\end{tabular}

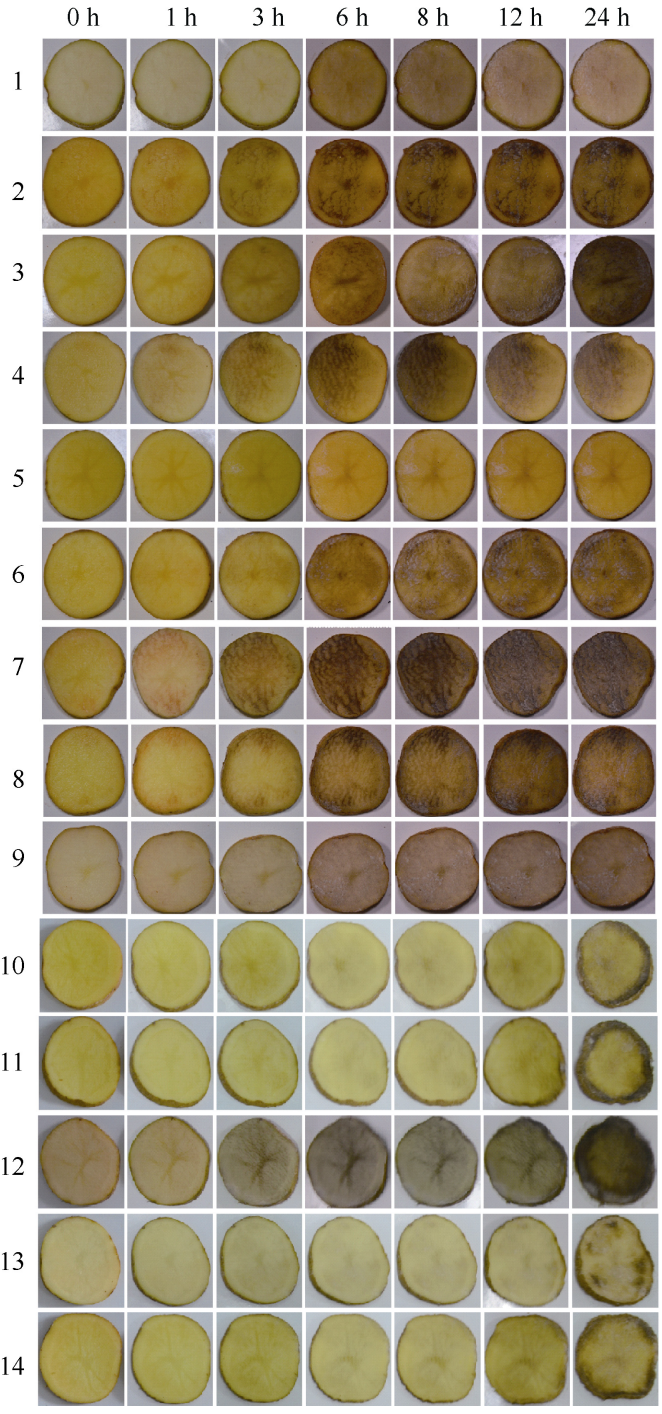

图 3 马铃薯品种(系)切片的褐变表型图

Fig. 3 Browning phenotype of slices of potato varieties (lines)

各材料的编号同表 1。Numbers of materials and the same as those given in Table 1.

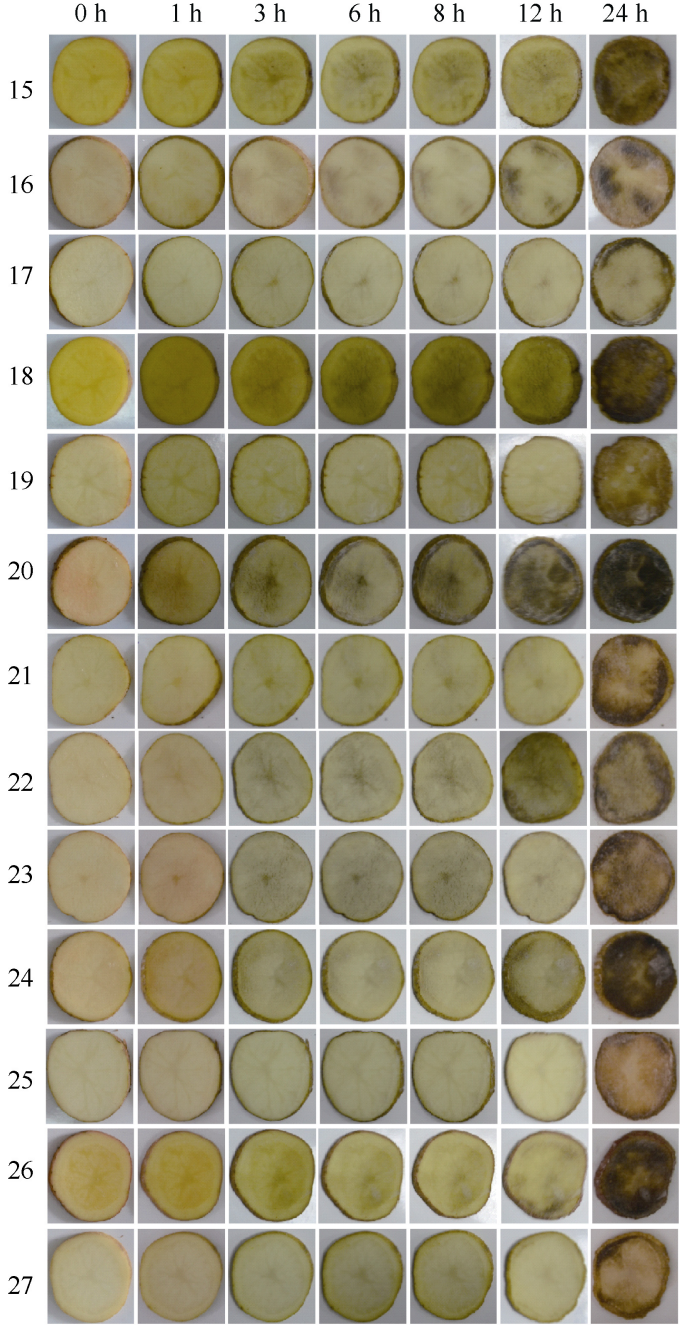




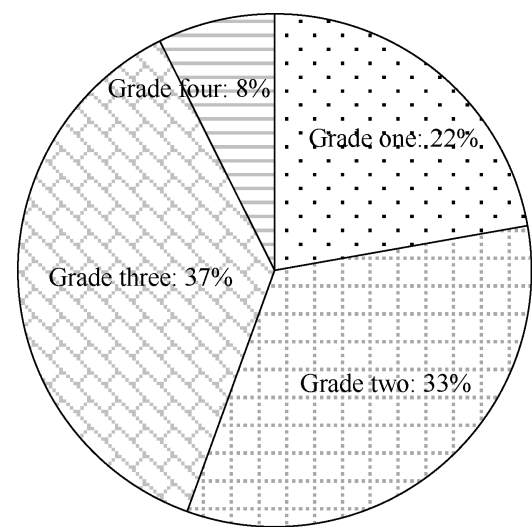

图 4 马铃薯品种(系)切片的褐变指数

Fig. 4 Browning index of slices of potato varieties (lines)

褐变指数最低, 为 $13 \%$; 褐变指数在 $25 \% \sim 50 \%$ 以内 的材料有 9 份, 占试验材料的 $33 \%$; 褐变指数在 $50 \% \sim 75 \%$ 以内的材料有 10 份，占试验材料的 $37 \%$; 褐变指数在 $75 \%$ 以上的材料有 2 份, 分别是春薯 6 号和费乌瑞它。

将 $24 \mathrm{~h}$ 总褐变指数在 $50 \%$ 以内，且开始发生褐 变时间于切片 $1 \mathrm{~h}$ 后作为抗褐变马铃薯预选材料, 共选出 15 份(表 3)。

\section{5 马铃薯品种(系)的煮后变褐}

煮后变褐 (ACD) 对于鲜食或加工都是一个重 要参考指标。通过观察发现延薯 4 号和 14018-142 $\square$ Grade one: 09306-82, Yanshu 4, 19-1, 13041-52, Chunshu 3 , Q8

G Grade two: 14018-142, 12-1, Atlantic, BF006, Lishu 6, Chunshu 5, Longshu 12, Weiyu 5, Zaodabai.

$\square$ Grade three: Dongnong 310, 15-1, Yunshu 505, Qianyu 8, Longshu 6, Qingshu 9, Minshu 1, 09001-136, Longshu 4, Zhongshu 5.

$\square$ Grade four: Chunshu 6, Favorita.

表 3 抗褐变马铃薯预选材料的褐变指数

Table 3 Browning index in anti-browning potato varieties (lines)

\begin{tabular}{|c|c|c|c|c|c|c|c|c|}
\hline \multirow{2}{*}{$\begin{array}{l}\text { 编号 } \\
\text { No. }\end{array}$} & \multirow{2}{*}{$\begin{array}{c}\text { 品种(系) } \\
\text { Variety (line) }\end{array}$} & \multirow{2}{*}{$\begin{array}{c}\text { 初始褐变时间 } \\
\text { Initial browning } \\
\text { time (h) }\end{array}$} & \multicolumn{6}{|c|}{ 褐变指数 Browning index (\%) } \\
\hline & & & $3 \mathrm{~h}$ & $6 \mathrm{~h}$ & $8 \mathrm{~h}$ & $12 \mathrm{~h}$ & $24 \mathrm{~h}$ & $\begin{array}{c}\text { 平均 } \\
\text { Average }\end{array}$ \\
\hline 5 & 09306-82 & 6 & 5 & 15 & 15 & 15 & 15 & 13 \\
\hline 11 & 延薯 4 号 Yanshu 4 & 8 & 10 & 10 & 10 & 25 & 40 & 19 \\
\hline 19 & $13041-52$ & 12 & 5 & 5 & 5 & 10 & 75 & 20 \\
\hline 13 & $12-1$ & 6 & 15 & 15 & 25 & 25 & 25 & 21 \\
\hline 10 & $19-1$ & 4 & 15 & 15 & 15 & 25 & 40 & 22 \\
\hline 17 & 春薯 3 号 Chunshu 3 & 8 & 10 & 10 & 20 & 15 & 55 & 22 \\
\hline 25 & Q8 & 12 & 15 & 15 & 15 & 5 & 70 & 24 \\
\hline 14 & $14018-142$ & 5 & 10 & 15 & 15 & 35 & 55 & 26 \\
\hline 1 & 大西洋 Atlantic & 6 & 10 & 40 & 45 & 35 & 20 & 30 \\
\hline 21 & BF006 & 6 & 10 & 25 & 25 & 25 & 65 & 30 \\
\hline 27 & 丽薯 6 号 Lishu 6 & 12 & 15 & 25 & 25 & 20 & 65 & 30 \\
\hline 24 & 春薯 5 号 Chunshu 5 & 1 & 15 & 15 & 15 & 45 & 95 & 37 \\
\hline 9 & 龙薯 12 号 Longshu 12 & 6 & 10 & 55 & 55 & 55 & 55 & 46 \\
\hline 15 & 威芋 5 号 Weiyu 5 & 3 & 35 & 35 & 40 & 40 & 85 & 47 \\
\hline 23 & 早大白 Zaodabai & 1 & 35 & 45 & 45 & 40 & 70 & 47 \\
\hline
\end{tabular}




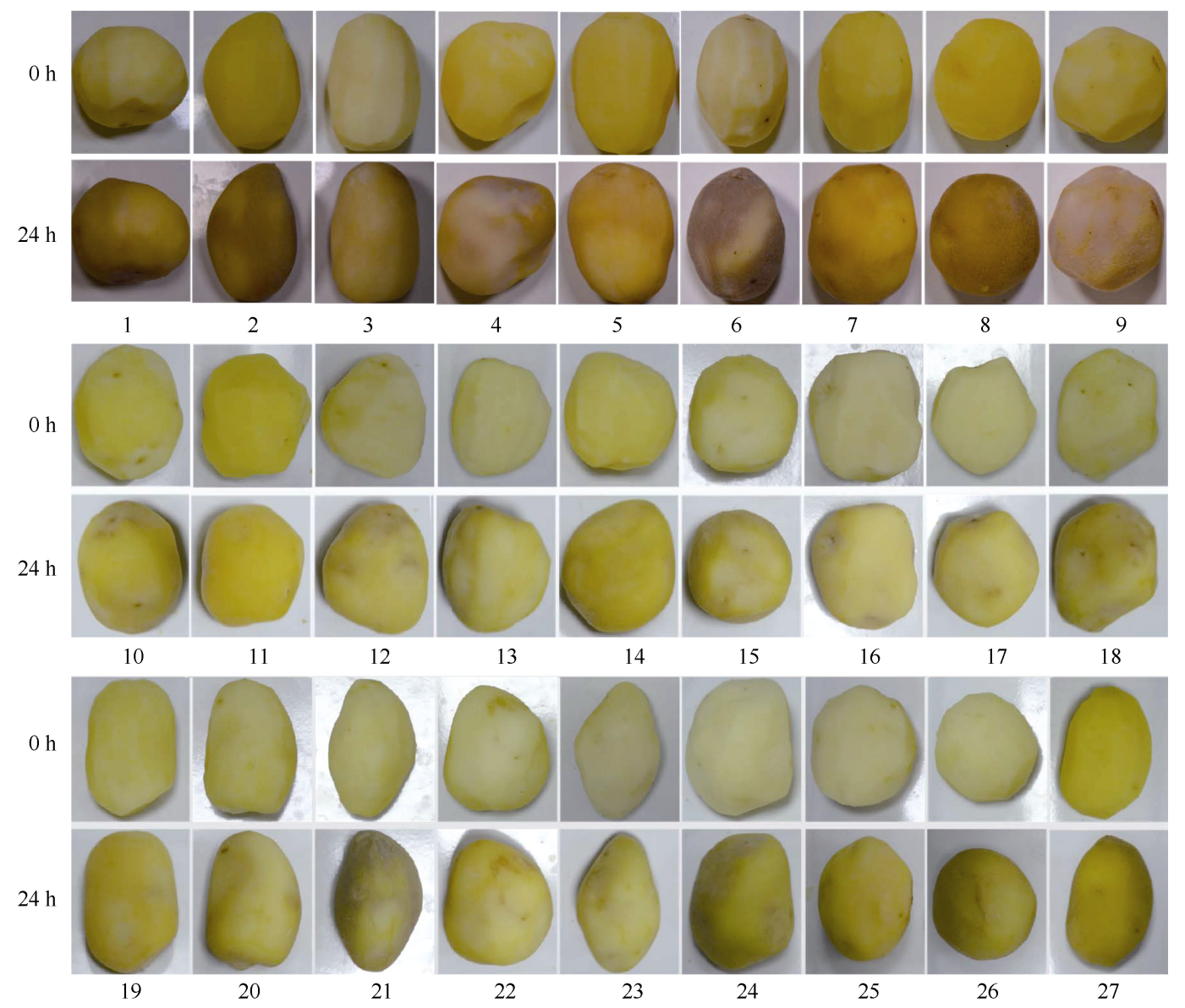

图 5 马铃薯品种(系)的煮后变褐

\section{Fig. 5 Browning of potato varieties (lines) after cooking}

各材料的编号同表 1。Numbers of materials and the same as those given in Table 1.

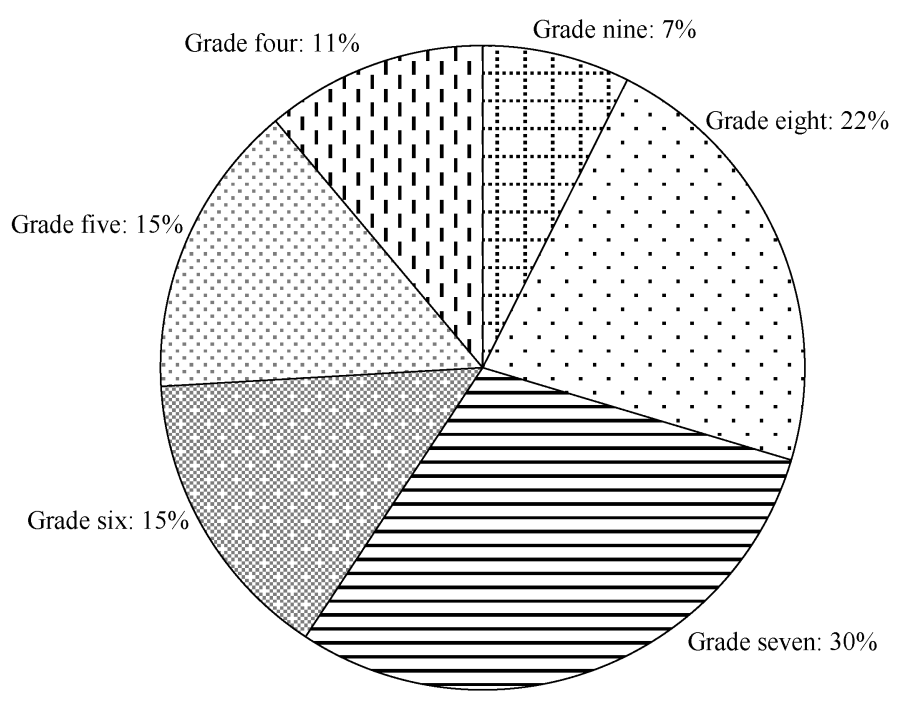

Grade nine: Yanshu 4, 14018-142.

$\square$ Grade eight: 09306-82, 12-1, Dongnong 310, Chunshu 3, 13041-52, Yunshu 505.

曰 Grade seven: Favorita, 19-1, Chunshu 6,

Weiyu 5, Longshu 4, 15-1, Zaodabai,Q8

圆 Grade six: 09001-136, Longshu 12, Qingshu 9 Lishu 6.

Grade five: Atlantic, Zhongshu 5, Longshu 6, Chunshu 5.

ㅁ Grade four: Qianyu 8, Minshu 1, BF006.

图 6 马铃薯品种(系)的煮后变褐分级

Fig. 6 Browning grading of potato varieties (lines) after cooking

\section{6 马铃薯品种(系)抗褐变能力综合评价}

篮选抗褐变马铃薯材料时需要多方面考虑, 单 一指标好的不一定具有较好的抗褐变能力, 例如
09001-136 和威芋 5 号 PPO 活性都较低, 但是切片褐 变早或者褐变程度重。这要求抗褐变马铃薯材料其 PPO 活性、褐变指数和褐变强度都低, 在蒸煮过后 
不发生严重褐变。对本研究中的 4 个褐变相关指标, 应在满足褐变指数和煮后变褐评价的前提下, 再结 合 PPO 活性和褐变强度对测定结果综合分析，因褐 变指数和煮后变褐比较直观体现褐变情况, 并且观 察的时间点多或者褐变时间充分。

27 份马铃薯材料可分为 4 种类型, 第 1 类高抗 褐变, 包括 09306-82 和 13041-52, 它们 4 项指标均 能满足抗褐变材料的要求; 第 2 类抗褐变材料, 包 括延薯 4 号、Q8、12-1、春薯 3 号、19-1、14018-142、 大西洋、龙薯 12 号、威芋 5 号、丽薯 6 号、春薯 5 号和早大白, 这类材料在褐变相关指标测定中存在 个别明显不足; 第 3 类中度褐变材料包括 09001-136、中薯 5 号、龙薯 4 号、东农 310、青薯 9 号、15-1、云薯 505、BF006、闽薯 1 号和黔芋 8 号, 这类材料抗褐变能力较弱; 第 4 类是易褐变材 料, 包括春薯 6 号和费乌瑞它, 它们反映出不具有 抗褐变能力或抗褐变能力很差。

\section{3 讨论}

马铃薯褐变包括酶促褐变与非酶褐变, 在现实 生活中, 有时两者是同时存在的, 因此在抗褐变材 料篮选中应充分考虑。酶促褐变的发生需要氧化酶、 酚类物质和氧气, 酚类物质作为底物在马铃薯块茎 内分布广、种类多, 不易确定与褐变的直接关系 ${ }^{[23]}$, 因此, 本研究将 PPO 活性作为一个抗褐变马铃薯篮 选指标。褐变强度和褐变指数测定在酶促褐变研究 中存在互补性, 褐变指数是对块茎切片, 其褐变程 度与切面的 PPO 和酚类物质区域化破坏程度有关, 褐变强度则是打破 PPO 和酚类物质的区域化分布, 使其充分相遇发生褐变反应 ${ }^{[21]}$ 。煮后变褐属于非酶 褐变 ${ }^{[23]}$, 这个指标对马铃薯抗褐变篮选研究很有意 义, 使研究更加全面, 结果更有说服力。褐变指数和 煮后变褐这 2 项指标有分级标准, 但 PPO 活性和褐 变强度并没有统一的分类标准, 本研究将 PPO 活性 在 $300 \mathrm{U} \mathrm{g}^{-1}$ 以内作为抗褐变预选材料, 是根据这些 材料整体变化趋势, 因为在 $300 \mathrm{U} \mathrm{g}^{-1}$ 以内的材料 PPO 增加趋势较缓慢, 考虑到 PPO 活性越高越易发 生褐变, 故将 $300 \mathrm{U} \mathrm{g}^{-1}$ 设为一个预选标准。研究中 09001-136 和威芋 5号 PPO 活性都较低, 但是观察切 片褐变表型发现褐变发生早或者褐变程度重, 这可 能与 2 份材料的酚类物质含量或者其他氧化酶的含 量有关, 酚类物质含量对酶促褐变的发生程度影响 很大，此外也有其他酶类催化酚类物质发生氧化反
应, 如过氧化物酶等。褐变强度是参考王海艳等 ${ }^{[24]}$ 篮选标准确定的, 因为初始褐变强度对于褐变有着 重要影响, 终止褐变值的测定是经过了 $24 \mathrm{~h}$, 故将 其篮选标准中初始值 0.20 改为了 0.15 , 变化值 0.15 改为了 0.20 。本研究将观察法和数据测定结合起来 是一种较可靠的马铃薯抗褐变鉴定篮选方法。

\section{4 结论}

通过对 27 份马铃薯材料 4 个褐变相关指标的测 定, 将其分为高抗褐变、抗褐变、中度褐变和易褐 变材料 4 种类型。高抗褐变和抗褐变材料可以为马 铃薯净菜加工提供品种支撑, 是鲜食抗褐变马铃薯 育种材料, 其中高抗褐变材料 09306-82 和 13041-52 是高代品系，完成相关试验后可进行新品种登记并 推广, 易褐变材料春薯 6 号和费乌瑞它抗褐变能力 差, 可用于马铃薯褐变机制的基础性研究。

\section{References}

[1] 胡军, 段绍光, 徐建飞, 市春松, 李广存, 庞万福, 金黎平. 马 铃薯块茎褐变相关研究进展. 见: 屈冬玉, 陈伊里主编. 马铃 薯产业与脱贫攻坚(2018). 哈尔滨：哈尔滨地图出版社, 2018. pp 119-123.

Hu J, Duan S G, Xu J F, Bian C S, Li G C, Pang W F, Jin L P. Research progress of potato tuber browning. In: Qu D Y, Chen Y L, eds. Potato Industry and Poverty Alleviation (2018). Harbin: Harbin Map Press, 2018. pp 119-123 (in Chinese).

[2] Shepherd L V T, Alexander C J, Hackett C A, McRae D, Sungurtas J A, Verrall S R, Morris J A, Hedley P E, Rockhold D, Belknap W, Davies H V. Impacts on the metabolome of down-regulating polyphenol oxidase in potato tubers. Trans Res, 2015, 24: 447-461.

[3] Chang Y L, Kagan L V, Antoni W J, Susan K B. Enzymatic browning in relation to phenolic compounds and polyphenoloxidase activity among various peach cultivars. J Agric Food Chem, 1990, 38: 99-101.

[4] Mcevily A J, Iyengar R, Otwell W S. Inhibition of enzymatic browning in foods and beverages. Crit Rev Food Sci, 1992, 32: 253-273.

[5] Keilin D, Mann T. Polyphenol oxidase purification, nature and properties. Proc Royal Soc London Series B, Biol Sci, 1938, 125: 187-204.

[6] Capitani C D, Carvalho A C L, Botelho P B, Carrapeiro M M, Castro I A. Synergism on antioxidant activity between natural compounds optimized by response surface methodology. Eur $J$ Lipid Sci Technol, 2009, 111: 1100-1110.

[7] Taranto F, Pasqualone A, Mangini G, Tripodi P, Miazzi M M, Pavan S, Montemurro C. Polyphenol oxidases in crops: biochemical, physiological and genetic aspects. Int J Mol Sci, 2017, 18: 377 .

[8] 赵欣, 周婧, 陈湘宁, 许丽, 杨肖飞, 刘建新. OPP/CPP 膜中 不同气体比例对鲜切马铃薯片保鲜的影响. 食品工业科技, 
2017, 38(17): 207-211.

Zhao X, Zhou J, Chen X N, Xu L, Yang X F, Liu J X. Effect of different gas compositions in the OPP/CPP film on preservation of fresh-cut potato chips. Sci Technol Food Ind, 2017, 38(17): 207-211 (in Chinese with English abstract).

[9] Shahidi F, Ambigaipalan P. Phenolics and polyphenolics in foods, beverages and spices: Antioxidant activity and health effects. $J$ Funct Foods, 2015, 18: 820-897.

[10] 苏霞, 吴厚玖. 橙汁非酶褐变机制及控制措施. 食品与发酵工 业, 2011, 37(7): 148-151.

$\mathrm{Su} \mathrm{X}$, Wu H J. Non-enzymatic Browning of orange juice and its control measures. Food Ferment Ind, 2011, 37(7): 148-151 (in Chinese).

[11] Mishra B B, Gautam S, Sharma A. Browning of fresh-cut eggplant: impact of cutting and storage. Postharvest Biol Technol, 2012, 67: 44-51.

[12] 姜莉, 林长彬, 张明兰, 张洪福, 徐怀德. 超高压对马铃薯多 酚氧化酶和过氧化物酶的钝化研究. 食品工业, 2013, (1): 141-143.

Jiang L, Lin C B, Zhang M L, Zhang H F, Xu H D. Study on deactivation of ultra-high voltage to the potato polyphenoloxidase and the peroxide enzyme. Food Ind, 2013, (1): 141-143 (in Chinese with English abstract).

[13] Siddiq M, Sinha N K, Cash J N. Characterization of polyphenol oxidase from Stanley plums. J Food Sci, 1992, 57: 1177-1179.

[14] Waltz E. USDA approves next-generation GM potato. Nat Biotechnol, 2015, 33: 12-13.

[15] Murata M, Nishimura M, Murai N, Haruta M, Homma S, Itoh Y. A transgenic apple callus showing reduced polyphenol oxidase activity and lower browning potential. Biosci Biotechnol Biochem, 2001, 65: 383-388.

[16] Ali H M, El-Gizawy A M, El-Bassiouny R E, Saleh M A. The role of various amino acids in enzymatic browning process in potato tubers, and identifying the browning products. Food Chem, 2016, 192: 879-885.

[17] Cantos E, Tudela J A, Gil M I, Espinet J C. Phenolic compounds and related enzymes are not rate-limiting in browning development of fresh-cut potatoes. J Agric Food Chem, 2002, 50: 3015-3023.

[18] Cabezas-Serrano A B, Amodio M L, Cornacchia R, Rinaldi R,
Colelli G. Suitability of five different potato cultivars (Solanum tuberosum L.) to be processed as fresh-cut products. Postharvest Biol Technol, 2009, 53: 138-144.

[19] 庞学群, 张昭其. 防褐处理对切分马蹄、马铃薯低温諚藏期间 褐变的影响. 食品科学, 2002, 23(4): 126-129.

Pang X Q, Zhang Z Q. Effects of anti-browning treatments on Browning of split-hoof and potato during low temperature storage. Food Sci, 2002, 23(4): 126-129 (in Chinese with English abstract).

[20] 李山云, 隋启君, 白建明, 杨琼芬, 李文鹏. 抗机械损伤褐变 马铃薯品种(系)的篮选. 中国马铃薯, 2010, 24(4): 193-196.

Li S Y, Sui Q J, Bai J M, Yang Q F, Li W P. Screening of potato (Solanum tuberosum L.) cultivars (lines) for browning tolerance responding to mechanical stress. Chin Potato J, 2010, 24(4): 193-196 (in Chinese with English abstract).

[21] 王清, 黄惠英, 马文芳, 王蒂. 反义 PPO 基因对马铃薯块茎褐 化的影响. 作物学报, 2007, 33: 1822-1827.

Wang Q, Huang H Y, Ma W F, Wang D. Effect of anti-sense PPO gene on the tuber-browning of Solanum tuberosum L. Acta Agron Sin, 2007, 33: 1822-1827 (in Chinese with English abstract).

[22] 王唯俊. 荷兰马铃薯高世代无性系产量及品质分析. 东北农 业大学硕士学位论文, 黑龙江哈尔滨, 2011.

Wang W J. Analysis of Agronomic and Quality Traits in Advanced Dutch Potato Clones. MS Thesis of Northeast Agricultural University, Harbin, Heilongjiang, China, 2011 (in Chinese with English abstract).

[23] 王丽, 王万兴, 索海翠, 胡新喜, 秦玉芝, 曾璐, 李小波, 熊兴 耀. 马铃薯块茎酶促褐变及与相关生理指标的关系. 园艺学 报, 2019, 46: 1519-1530.

Wang L, Wang W X, Suo H C, Hu X X, Qin Y Z, Zeng L, Li X B, Xiong X Y. The relationship between enzymatic browning and relevant physiological index of potato tubers. Acta Hortic Sin, 2019, 46: 1519-1530 (in Chinese with English abstract).

[24] 王海艳, 王立春, 李凤云, 田国奎, 娄树宝, 李成君, 郝智 勇. 马铃薯抗褐变种质资源的鉴定与篮选. 植物遗传资源 学报, 2018, 19: 263-270.

Wang H Y, Wang L C, Li F Y, Tian G K, Lou S B, Li C J, Hao $\mathrm{Z}$ Y. Identification and selection for potato anti-browning germplasm resources. J Plant Genetic Resour, 2018, 19: 263-270 (in Chinese with English abstract). 